\title{
Value creation and value capture for Al business model innovation: a three-phase process framework
}

\author{
Josef Åström ${ }^{1} \cdot$ Wiebke Reim $^{1}$ (D) . Vinit Parida ${ }^{1}$
}

Received: 28 May 2021 / Accepted: 1 January 2022 / Published online: 20 January 2022

(c) The Author(s) 2022

\begin{abstract}
The rise of AI technologies is generating novel opportunities for companies to create additional value for their customers by applying a proactive approach, managing uncertainty, and thus improving cost efficiency and increasing revenue. However, AI technology capabilities are not enough — companies need to understand how the technology can be commercialized through appropriate AI business model innovation. When emerging technologies are introduced, business-model concepts often need to be significantly altered. This is necessary to fully capitalize on disruptive technologies because it is just as important to innovate the business model as it is to build advanced technology solutions. Therefore, the purpose of this study is to explain how AI providers align value-creation and value-capture dimensions in order to develop commercially viable AI business models. To fulfill our stated purpose, this study has adopted an inductive and exploratory single case-study approach centered on a market-leading provider of AI-related services. The findings are consolidated into a process framework that explicitly illustrates the key activities that companies need to perform concerning value creation and value capture for AI business model innovation and commercialization. The framework explains that AI providers need to follow three phases-namely, identifying prerequisites for AI value creation, matching value capture mechanisms, and developing AI business model offer. We also find that AI providers need to test and develop multiple AI business models and operate them simultaneously to ensure commercial success.
\end{abstract}

Keywords Business models · Artificial intelligence (AI) - Value creation · Value capture $\cdot$ Process framework

Mathematics Subject Classification 91

Wiebke Reim

wiebke.reim@1tu.se

1 Entrepreneurship and Innovation, Luleå University of Technology, Luleå, Sweden 


\section{Introduction}

Artificial intelligence (AI) is often referred to as a technology that facilitates the creation of additional value for customers. This stems from the advances in AI techniques that allow us to mimic cognitive behavior and automate the processes of identifying and solving complex problems (Lee et al. 2019; Zhuang et al. 2017). According to Lee et al. (2019), AI offer opportunities to improve operational efficiency and accelerate innovation by deriving insights from large data sets and predicting unexpected events, which is a development of considerable interest across many diverse industries. However, Brock and Von Wangenheim (2019) stress that the successful integration of AI applications is difficult to achieve because, in order to develop certain capabilities and resources, major investments and long development cycles are required. This will strengthen the opportunities for AI providers to become specialists in the subject and offer AI solutions to the industry.

The rise of AI gives providers the opportunity to create additional value by applying a proactive approach, managing uncertainty, and thus improving cost efficiency and increasing revenue (Cockburn et al. 2018; Laudien and Pesch 2019). However, to capitalize on the technology, it is important to understand how the technology can be commercialized by employing an appropriate business model (Bouncken et al. 2021). According to Valter et al. (2018), previous research falls short in understanding how firms successfully operationalize AI solutions through their business models, particularly with regard to technological advancements in the AI sphere. From a practical point view, this finds support in the Artificial Intelligence Global Executive Study and Research Report by Ransbotham et al. (2019) where it is shown that $40 \%$ of their respondents conceded that significant investment did not deliver the sought-after business gains.

To understand this, business models can be described as "a mediating construct between technology and economic value" (Chesbrough and Rosenbloom 2002) and can be divided into two important functions: value-creation mechanisms and value-capture mechanisms (Chesbrough 2007). In other words, the business model describes a set of activities that aims to satisfy the final customer and sets out how the operating firm intends to capture economic return from the defined activities. Thus, when emerging technologies are introduced, business model concepts need to be transformed in order to fully capitalize on disruptive technologies. This is because it is just as important to innovate the business model as it is to build advanced, technical solutions (Chesbrough 2007; Muhic and Bengtsson 2021). With that said, recent technical progress in the AI field and the lack of understanding of how technical progress can be transformed into business gains make further research essential to enable the technology to be successfully applied. This is supported by Lee et al. (2019) who mention that further research is vital in order to understand how AI solutions can be commercialized using different business-model archetypes. In addition, Nylund et al. (2020) assert the need for future research on how value can be captured when commercializing technological innovation in the context of industry disruption. 
With regard to the literature gaps identified and the practical challenges that abound, our study's purpose is to explain how AI providers align value-creation and value-capture dimensions in order to develop commercially viable AI business models. More specifically, our framework illustrates activities that build the necessary dimensions and increase understanding of how value creation and value capture interact. The research purpose is served by employing a single-case study of a market-leading AI provider in the telecoms industry. From a theoretical point of view, this study extends our understanding of AI and how value-creation and valuecapture mechanisms relate to each other. Additionally, the framework carries certain practical implications for AI providers who seek to commercialize AI techniques through the deployment of appropriate business models. The framework presented, and the activities associated with it, further increase understanding of how AI providers bring AI-related opportunities to fruition.

The following section of the paper presents the theoretical background, and the third section describes the method used including data collection and analysis. In the fourth section, the empirical results and our framework is presented. The final section summarizes the theoretical contributions, managerial implications and areas for future research.

\section{Theoretical background}

\subsection{Artificial intelligence and business application}

Over recent years, AI has created fascination and widespread interest. It can be defined as machines' ability to mimic human-like behavior through processes such as learning, reasoning and self-correction (Kok et al. 2009; Lee et al. 2019; Zhuang et al. 2017). Moreover, AI can be explained by referencing four requirementsnamely, natural language processing, knowledge representation, automated reasoning, and machine learning (Kok et al. 2009). However, when we look at AI in terms of its current ability to fundamentally transform businesses, we should be careful not to overstate what it is capable of at present. AI technology cannot be considered mature enough to replace all aspects of human cognition (Agrawal et al. 2019; Kakatkar et al. 2019). As mentioned by LeCun et al. (2015), further progress in the field of AI will emerge from systems that combine learning algorithms with complex reasoning, meaning that the collected knowledge of systems can be applied in different settings.

The practical meaning of AI and its impact can be understood on the level at which reasoning, and machine learning techniques are combined. Kaplan and Haenlein (2019) communicated this insight through three maturity levels of AI, with an explanation of the potential business impact of each. The first maturity level includes only machine learning, and its operational impact is therefore limited to specific areas of application. Lessons learned from a specific setting cannot be applied to new areas, and dramatic changes in input parameters will decrease the functionality of the algorithms. The second maturity level extends the operational impact to include several areas of application, meaning that learning can be applied to new, 
unexplored settings without human intervention. This is therefore where simple reasoning comes in, allowing an expanded area of powerful machine learning algorithms to be used. Lastly, the third level of maturity encompasses a fully selfconscious system with the ability to interconnect its creativity and general wisdom with operational problems in any area. In addition, the third maturity level of AI has the ability to outperform humans in any area. That is to say, it can out-compete all aspects of human cognition, which will ultimately make humans redundant (Kaplan and Haenlein 2019).

Due to its technical dynamism, artificial intelligence is considered by many to be a general purpose technology (GPT) in the digital era (Bresnahan and Trajtenberg 1995; Brynjolfsson et al. 2017; Cockburn et al. 2018). GPTs are constructed in a way that makes them applicable to a large number of different customers and generates productivity gains throughout the economy (Gambardella and McGahan 2010). An example of a GPT can be found in electricity, where all industries and firms are dependent on the electricity infrastructure in order to perform internal operations. Electricity providers are thus able to capture a small portion of value out of a large customer base by standardizing the new technology. Furthermore, Gambardella and McGahan (2010) argue that introducing GPTs creates intermediate technology markets where downstream enterprises are provided with the required resources and capabilities needed to capitalize on the technology. Therefore, the industry structure and how it is affected by AI as a GPT should be considered when aiming to capture value through the provision of AI-related solutions. The view of AI as a GPT (Brynjolfsson et al. 2017; Cockburn et al. 2018) suggests that intermediate technology firms will position themselves as AI providers, whose purpose is to develop the resources and capabilities required to develop AI-related solutions (Gambardella and McGahan 2010).

\subsection{Value-creation opportunities through Al integration}

Value creation is often referred to as an essential function of the business model because it forms the basis for satisfying customer interests (Chesbrough and Rosenbloom 2002; Chesbrough 2007; Zhuang et al. 2017). It is defined by the series of activities needed to create a product or service that aims to solve certain problems and meet customer expectations (Chesbrough 2007). Therefore, it stems from customer needs and how technology can be applied to solve identified customer issues through a set of activities. Furthermore, the value-creation function describes how resources and capabilities should be allocated in order to create the value desired within the value creation network, which consists of firms cooperating to enhance the value-creation function (Barney 1999; Kraus et al. 2019).

Value-creation processes derived from AI can be divided into different categories-namely, (i) activities related to increased efficiency and cost reduction (Agrawal et al. 2019), and (ii) activities related to revenue and growth (Cockburn et al. 2018). Activities related to increased efficiency and cost reduction aim to improve and refine already existing operations, such as maintenance and daily production processes (Agrawal et al. 2019). Hence, the operation's output will be the 
same and the customer will not witness any revolutionary changes in the perceived value. However, cost efficiency and time spent on certain activities related to the operation will significantly improve. For instance, Agrawal et al. (2019) direct the focus on benefits from predictability and its impact on labor. They argue that AI can substitute elements of the decision processes with digitized solutions, which will reduce the need for repetitive work tasks and thus deliver cost savings. However, Cockburn et al. (2018) argue that the potential of AI goes further than cost savings and increased efficiency into the domains of revenue and growth.

Activities related to revenue and growth mean that, from recent advancements in the technology, value can be created through AI's ability to support decisions and improve outcomes (Cockburn et al. 2018). This essentially means that AI algorithms are fed data, and information is generated that was not previously available because AI has developed the capability to solve complex problems and provide insights with higher accuracy. Information gaps are thus filled with information that reflects reality to a greater extent. This further enables humans and technology to undertake actions that are supported with more accurate information. From a business perspective, support from AI enables managers to make more accurate decisions under conditions of uncertainty, thereby complementing the decision processes and improving outcomes. Plastino and Purdy (2018) further argue that AI facilitates revenue growth by accelerating innovation and developing new solutions that render new revenue streams. For instance, in the domain of drug development, Berg Health began to monitor trillions of data points derived from cancerous and non-cancerous cells, furnishing insights that led to the development of new cancer-fighting drugs (Thomas et al. 2016). This entailed not only lower costs in developing new drugs but it also brought a new level of innovation that could not be achieved by human intervention alone.

However, AI should not be considered an innovation that creates value on a stand-alone basis. Rather, Pisano and Teece (2007) argue that every innovation requires complementary products, technologies, or services to fulfill its purpose. For instance, every mobile phone need connectivity networks; airlines need airports; and hardware needs software. Thus, the innovation must be embedded with the complementary product, technology, or service in order to create value for the customer. If the firm lacks capabilities and/or resources to deliver the complementary offerings, its ability to create value might be limited. It is therefore important to understand how AI creates value and then to make sure that complementary products, technologies, and services are available within the value-creation network. Our limited understanding of AI applications and how value is created and how it is captured serves to highlight the importance of more research.

\subsection{Value capture opportunities through Al integration}

In this study, value capture is defined as the mechanisms that make sure that an economic return from value creation and that profits are shared throughout the valuecreation network (Sjödin et al. 2020). Hence, value-capture mechanisms build on the provider's role in the value-creation network and ensure that profits are fairly 
distributed throughout the value-creation network. More specifically, Sjödin et al. (2020) point out that governance mechanisms and legal agreements (e.g., contracts) play a substantial role in a firm's ability to capture value. Legal agreements increase transparency because they hold both customers and providers responsible for certain activities during the contract period. This is an issue of substantial importance because it has a direct impact on each party's performance, and subsequently it has a bearing on the ability to capture value.

Moreover, Gassmann et al. (2013) argue that pricing models constitute important mechanisms in the value-capture sphere because they convey the relationship between cost structures and the revenue mechanisms applied. They demonstrate also how the provider aims to sustain a profitable relationship with the customer. Consequently, this paper will investigate pricing models as the core element in value capture and explore how contractual agreements facilitate or hinder value capture when AI is introduced. Contractual agreements can therefore be understood as an extension of value propositions and pricing models, which formulate the basis on which value can be captured. However, as Gambardella and McGahan (2010) have attested, it is not enough to understand just single interrelationships between providers and customers when considering value capture opportunities in the context of disruptive innovations. They argue that businesses need to situate disruptive innovation and its impact on value capture in the broader perspective of AI and its overarching impact on industries.

\subsubsection{Value capture through pricing models}

According to Liozu et al. (2012), pricing models can be divided into two main categories - cost-based pricing models and value-based pricing models. The cost-based pricing model is seen as the dominant model in the pricing domain. It builds on the costs required to obtain the value created. Subsequently, a margin is added to ensure profitability for the provider. This margin is often determined on the basis of the solution's uniqueness in relation to competitors' available solutions in the marketplace (Liozu et al. 2012). Hence, cost efficiency becomes a central aspect when deploying the cost-based pricing model because lower cost equals a more attractive price in the marketplace. Cost-based pricing models are preferable given that the value propositions underpinning them are readily understandable for both customers and providers - costs are relatively easy to measure, and providers can easily communicate how value is created through their offerings (Hinterhuber 2008). However, despite their benefits, pure cost-based pricing models are seen as problematic because they fundamentally ignore the customer's perceived value and diminish the provider's incentives to undertake improvement and initiate innovation (Simon et al. 2003). Due to these limitations in cost-based pricing models, both researchers and practitioners have been shifting their focus to value-based pricing models (Simon et al. 2003; Hinterhuber 2008). This is readily apparent in the development of pricing models for software applications and information goods (e.g., AI offerings) because the marginal costs are close to zero (Choi et al. 2010).

Unlike the cost-based pricing model, the value-based approach builds on the value perceived by the customer and, therefore, it pays less attention to the costs 
required when delivering the value. According to Hinterhuber (2008), the two most profound challenges facing value-based pricing models are linked to value assessment and value communication. Here, the success rate of the value-based pricing model is more dependent on the provider's ability to understand and quantify the value created for the customer and then, to communicate the value created to the customer through a strong value proposition. With that said, both the cost-based approach and the value-based approach are linked to different benefits and risks. However, it is not known how AI providers relate to the two models. Nor is it understood to what degree value-based pricing models prove to be suitable when AI is introduced. This clearly highlights the need for further investigation.

\subsubsection{Value capture through contracts}

Beside selection of suitable pricing models, providers can commit to different commercial arrangements affecting how value is captured by the provider. These arrangements are further communicated to the customer through contracts. The contract acts as a mediator between provider and customer because it states how, and under which conditions, created value can be captured (Sjödin et al. 2020). In this section, two different contracts will be discussed. The first contract category, referred to as the outcome-based contract, is the most advanced offering because it provides a more comprehensive solution. Rolls-Royce and the "power-by-the-hour" concept is a classic example of delivering performance, or outcome, using a subscription model based on hours of operation rather than selling the actual engines or complementary add-on services to the customer. Therefore, in outcome-based contracts, providers receive compensation for the outcome or performance delivered, rather than supplying single add-on services or service agreements. Many firms are moving to outcome-based business models in order to create and capture more value from the technology (Visnjic et al. 2017). This type of model offers the potential to create added value using AI applications by boosting the opportunities that providers have to offer process outcomes rather than single products or services. Such offerings, however, require close collaboration between provider and customer (Sjödin et al. 2020), and outcome-based contracts need to share "the gain and the pain" between the parties involved (Hou and Neely 2018). To ensure that value is apportioned fairly, Sjödin et al. (2020) argue that the providers offering outcome-based contracts must evaluate the profit potential, define performance indicators, maintain incentive structures, and design an equitable profit formula.

The second contract category-the licensing model-is considered to be a default mechanism for value capture when offering GPTs (e.g., AI), according to Teece (2018). This is further supported by Moeen and Agarwal (2017) who contend that licensing models enable value capture from a greater number of sources with little to no effort on the part of the providing firm. Therefore, in contrast to the outcome-based contract, the licensing model offers the providing firm additional revenue streams for essentially the same workload. In addition, Moeen and Agarwal (2017) argue that the licensing model offers the ability to reach customers outside the boundaries of the industry, and so the customer base must not be limited to the industry in which the providing firm is active. The licensing model, therefore, 
enables greater value to be captured from innovations that are already created by targeting a larger set of customers, and by transferring responsibility for how the innovation is used to the licensee. However, Teece (2018) identified major challenges for firms seeking to profit from innovation using the licensing model. For instance, GPTs tend to generate large spillover effects, which means the customer benefits from a substantial economic contribution whereas the providing firm gets to capture only a small proportion of the value created. This points to the importance of developing capabilities to protect innovations - in other words, procuring support from legal mechanisms (e.g. non-disclosure agreements, patents, trade secrecy) or ensuring ownership of complementary assets (e.g., brand, distribution channels) in order to secure profitability (Pisano and Teece 2007; Teece 2018).

However, when discussing AI as a GPT, it is not known how the value created should best be captured in order to fully capitalize on the technology. On the basis of the literature reviewed, providers seeking to capture the value created would appear to have a choice between an outcome-based contract, a licensing contract, or a combination of both when offering AI applications. This highlights the current gap in the literature and therefore, our study's purpose is to explain how AI providers align value-creation and value-capture dimensions in order to develop commercially viable AI business models.

\section{Method}

\subsection{Research approach and strategy}

In this study, an exploratory case-study approach was adopted to explain how AI providers align value-creation and value-capture dimensions in order to develop commercially viable AI business models. This is considered an appropriate strategy given the limited theoretical understanding of the activities that are required to successfully implement AI, with special regard to recent progress in the AI field. A single case study enabled a more in-depth understanding of AI's impact on businesses. The selected case company is a provider of telecommunication and network infrastructure including related services. The case company has more than 100000 employees and operates worldwide. Furthermore, the case company selection was based on three evaluated criteria. Firstly, the case company we selected provided AI solutions to their customers, which meant that it possessed a proper understanding of AI itself as well as the potential that the technology held for application to different areas. Secondly, the company possessed a large, global customer baseits offerings covered telecom provision for 1.5 billion end users, indicating that its AI offerings covered numerous customer segments. In consequence, it possessed a diverse portfolio of AI applications, which gave it a full understanding of AI and how it could be utilized in a wide range of different settings. Thirdly, it had garnered considerable experience from designing and executing AI-related offerings. Thus, its market understanding, its experience as a fully-fledged AI provider, and its expert knowledge of AI constituted the basis for augmenting the current literature with indepth insights into the business opportunities that flow from AI. 
In this study, primary data was acquired from 23 interviews. Interviewees were selected on the basis of the corporate positions they held and the knowledge they commanded on the subject. The interviews were conducted with senior managers responsible for service portfolios, business development, capability development, and key accounts, among others. The interviews were semi-structured, and the recordings were transcribed afterwards. Beside data collection using interviews, the interviewees were asked to share internal documents that were then reviewed in order to acquire a better understanding of the company's provision of AI-related solutions. However, secondary data-in terms of internal documents-were only used in an initial phase, and their primary purpose was to build a better understanding of the case company, its AI-related offerings, and its specific characteristics as a telecom provider. Results were reported back to the case company to obtain its validation.

This study adopted a three-step process to analyze the collected data, including (i) identifying first-order activities, (ii) identifying second-order activities, and (iii) identifying aggregated phases., According to Gioia et al. (2013), a three-step process is preferable when analyzing exploratory data because of the systematic features it incorporates that enable new concepts and ideas to be generated. The first step was to identify key activities mentioned by informants, without attempting to categorize the terms (Gioia et al. 2013). This step was done by two researchers independently first. Afterwards the codes where shared and discussed. Thus, all activities that covered the interconnections between value creation, value capture, and AI were taken into consideration. The identification of activities without categorization facilitated the traceability of the study's findings and made it possible to go back to the originating terms and understand them in their context. In order to successfully identify the first-order activities, all interviews were transcribed and read carefully. Secondly, the collected data were processed by seeking similarities and differences among the first-order activities identified, and thereafter interlinked activities were clustered into overarching activities (Gioia et al. 2013). In the third step, the secondorder activities identified were interlinked by creating aggregated themes. These themes constituted the third and most abstract level in the analysis. To ensure its trustworthiness, iteration between the three steps (i.e., first-order activities, secondorder activities, and third-order phases) were made to confirm the logic underpinning their connections. The three steps, and how they are interlinked, forms the data structure, which is depicted in Fig. 1.

\section{Results and analysis}

This section presents our results, based on the coding tree in Fig. 1. It starts with the activities needed to identify the prerequisites for AI. The second theme builds a bridge between the value-creation and the value-capture dimensions. To match value creation with value-capture mechanisms, the task at hand is to link value-creation opportunities with the targeted customer segment. Lastly, the third theme covers the development of AI business model offers and the means through which value is to be captured. Different value-creation opportunities mediate different approaches 


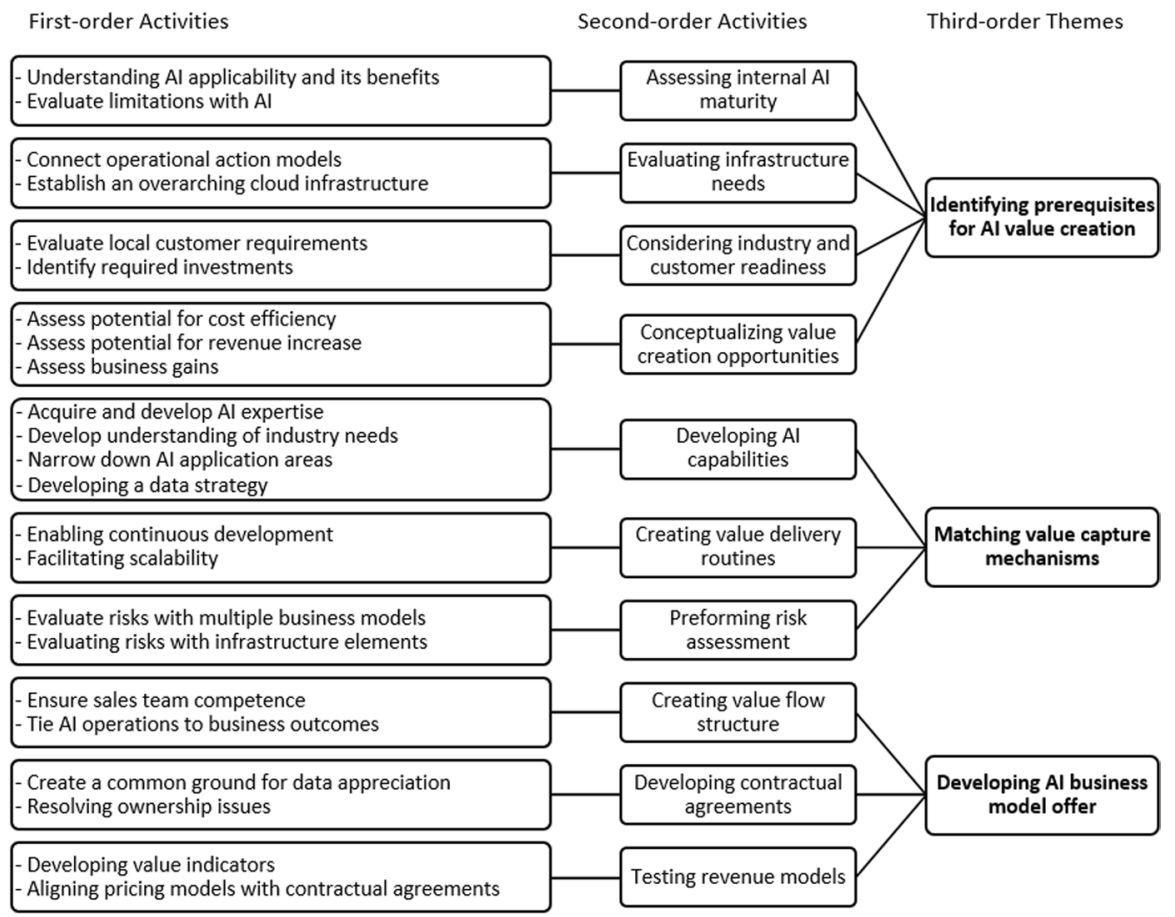

Fig. 1 Visualization of the data structure

in the design of pricing models and contractual agreements. The following section offers a detailed explanation of the empirical results.

\subsection{Identifying prerequisites for Al value creation}

The first third-order theme centers on the value-creation activities needed to establish a value-creation network possessing the capabilities to create value using AI. This network is further cultivated through four second-order activities, framed as assessing internal AI maturity, evaluating infrastructure needs, considering industry and customer readiness, and conceptualizing value creation opportunities. In this section, the four second-order activities will be further explained in consecutive order.

Assessing internal AI maturity. When discussing value creation, the informants highlighted the importance of understanding the technical aspects of AI. This requires the provider to obtain an understanding of the technical aspects, where it is applicable, and how it generates value. Ideally, this evaluation process will result in a detailed framework explicitly describing the technical progress of AI in accordance with Kaplan and Haenlein (2019) who describes the different maturity levels of AI. The enhanced technical understanding will further consolidate the foundations so that an environment can be built in which the benefits of AI can be realized. 
However, it is of fundamental importance to understand the limitations of AI technology as currently developed because this will have a direct impact on how value is created, as stated by the AI and Automation Development Lead:

... when something new comes in [to the system] and the AI is not trained to handle it, it will become a problem. Reasoning handles that because now we are combining information that you may have seen in the past from other domains, or external data sources, or $M L$.

Evaluating infrastructure needs. When the provider possesses a technical understanding of AI, it is then important to grasp the surrounding infrastructure elements that are needed to create the desired value. The informants noted that the main benefits of AI are derived from its ability to predict unexpected happenings and events that would otherwise trigger faults in the network. Output from the AI model can provide information on when, where, and how faults can be handled before they occur, offering the ability to apply a proactive approach to maintaining the network. However, the perceived customer value is dependent on how, when, and where actions are taken, meaning that the information provided by the AI model does not create value by itself. Rather, operational actions taken by the receiver of the information will decide whether, and to what extent, the fault can be handled, which in turn decides how the customer perceives value. Thus, a model that incorporates when, where, and how operational actions are taken, based on the information provided by the AI model, is required in order to create any value from the information provided by AI. This operational action model can involve humans or complementary techniques enabling automation, according to the Service Portfolio Manager in UK:

We say'Yeah, we predicted it', but if we don't take action, we will not create value, we now need to take it to the next step with automation, so using our interconnected platforms to enable our automation platform and having activities that we can do automatically off the back of these predictions is really the end game for us.

It is also important that the infrastructure is built from an overarching cloud solution, which enables learning in an environment that maximizes data inputs. According to the informants, the potential of AI increases if the algorithms are honed in a centralized environment where they can access larger amounts of data rather than in decentralized customer locations with limited data access. Therefore, there is a clear demand for an infrastructure that supports learning activities and thus value creation.

We are now going to be running our tools on a private cloud, we got the data on a private cloud, and effectively, we have to move the data from the customer's tools to our tools, to be able to leverage the benefits with automation and AI etcetera.

Considering industry and customer readiness. Throughout the interviews, the informants stressed the importance of industry readiness. This includes an 
appreciation of governmental regulations in different geographical areas, which affects how data can be utilized to create value through AI solutions. Governmental regulations relate mainly to end-subscriber information-for example. personal information on individuals and consumer patterns. Evaluation of these regulations is important in order to grasp the limitations placed on value-creation activities. However, regulations vary between geographical areas and, therefore, analysis on a decentralized basis is required. Furthermore, it is important to evaluate whether customers are able to obtain the required data and what their approaches to sharing data are. Generally, the customer's approach to data will have a direct impact on how and when it can be accessed, and ultimately on how the AI provider will be able to create value, which points to the importance of evaluating the interests of customers in this domain. Lastly, industry readiness involves the customer's approach to acting on insights provided by AI models. This means that customers must trust the information provided and subsequently implement changes in accordance with the insights imparted. However, this will likely require substantial investment or re-prioritized action plans, which can lead to customer resistance. As previously stated, the ultimate value created by AI is directly dependent on how the information provided is utilized, which reinforces the importance of assessing industry readiness.

Conceptualizing value creation opportunities. Finally, technical AI understanding should be translated into potential value-creation opportunities, with respect to prerequisites identified in the surrounding environment. This study proposes an assessment in three areas-namely, cost efficiency improvement, revenue increases, and business gains. Thus, the results of our study confirm that an AI operation supports cost efficiency through its ability to provide support for decisions by handling large and complex amounts of data. Insights provided by AI can thus be used to make informed decisions and subsequently reduce costs, whilst the process output remains the same. Additionally, this study demonstrates that information provided by AI can facilitate an increase in revenue by providing qualitative information that serves to improve the process output. The head of capabilities development at Alpha offered an example:

People at the lower job stage are doing quite repetitive work and shift work, so at the first step we view automation within that area where we would use AI to automate that work and clear away all that noise.

However, while insights provided by AI can have a direct impact on decisions on an operational level and thus directly impact cost efficiency and revenue increases, this study extends this view by showing that AI can provide higher-level insights that result in overarching business gains. This means that AI can deliver insights at both the operational and the business level, which requires different application areas and thus results in different values. Having said that, our study makes a distinction between increases in operational revenue and overarching business gains. The informants provided examples of numerous different business gains created by AI. However, increased awareness among actors in the value-creation network was mentioned as a fundamental business gain. This is particularly important given the increased volume of devices that need to be handled, the more diverse 
service requirements, and the increased complexity of network operations. Despite this change, AI provides the ability to correlate different data sources to identify and measure the value created and, thereby, increase awareness of how the network should be operated.

For the existing customers, we have dashboards so we can monetize what the SLA is, so we won't end up signing anything we can't, or need to keep. There will be many assessments to see, and it is going to help both operators and us. So, when it comes to SLAs, we will sign and being more aware than the earlier SLAs, aware of the value we are creating because we can see and define that. We are aware of the cost which we will enter because it will be our tools that we need to reinforce for a valid reason.

To sum up, evaluation of opportunities in the three value categories-namely, cost efficiency, revenue increases, and overarching business gains-is required in order to understand the value-creation dimension when AI is introduced. In addition, to confirm that the values identified are possible to achieve, it is necessary to iterate between the four second-order activities.

\subsection{Matching value capture mechanisms}

The second theme builds a bridge between the value-creation and value-capture dimensions, using the knowledge gained from the first phase as the basis. Unlike the first phase, this phase is more customer oriented; it aims to specify how and where values will be created, subsequently tying the identified opportunities to customer needs. The three second-order activities-namely, developing AI capabilities, creating value delivery routines, and performing risk assessment will be described in consecutive order.

Developing AI capabilities. Informants stated that the value-creation network must possess not only in-depth AI expertise and proper understanding of industry needs but also the ability to combine AI expertise and industry understanding when developing an AI offering. Given the identified prerequisites for an AI solution, the provider must define and develop the scope and breadth of the business and to which customer segment the AI solutions will be applied. Hence, in this stage, the provider must utilize its industry understanding to develop a conceptual framework that can be applied to potential AI application areas. In other words, AI expertise must be combined with industry understanding to further exploit the potential of AI. The purpose of this phase - namely, developing a competitive edge- is about tying value creation opportunities to actual customer needs and refining value-creation activities to the requirements of different customer segments. As the business development director in the US stated:

Operators around the globe have most data available, and they are the least innovative in terms of AI and ML worldwide. So, to understanding those structured data components, which includes understanding the performance counters, which is a decision tree mechanism. So, as we have the technical 
operations expertise and the telecom experience, we see that for each scenario which model works best.

However, when developing the competitive edge by blending AI expertise and industry understanding, it is important to fully exploit the providing organization's capacity for industry understanding. Informants commented that AI offerings are limited only by the providing organization's imagination and, therefore, the number of application areas will increase exponentially over time. Consequently, the conceptualization of potential AI usage areas ought not to be limited to traditional AI delivery mechanisms but rather should be spread across the organization in order to enable AI applications for all types of customers. The commercial manager in the US expressed it in these words:

There is one unique advantage on our radio side, we will have more knowledge radios than we do of our competitors. And, on the managed services side, we do operate everybody's network, so how do we blend those two and not cross any inappropriate borders, but how do we drive our advantage in operating networks?

All informants stated that data is of central importance when discussing AI solutions, because data exert a direct impact on the algorithm's output. Therefore, it is important to access large amounts of data in real time in order to enable value creation through AI. However, as previously affirmed, data usage is often limited by data sensitivity and customers' general resistance to sharing data. Therefore, it is important that the AI provider extends its AI focus area with a suitable data strategy that communicates why, when, and how data must be accessed in order to enable value creation through AI. Essentially, the provider must develop a clear strategy regarding how the data are used and where they are stored. This resistance to sharing data often occurs when the customer is unaware of how data will be used and who will be given access to the data. Additionally, the data strategy must communicate in what format the data will be accessed, and how it is transferred into readable format that the algorithms can handle.

$80 \%$ of the activity with machine learning is getting the data in the format that we can then learn from, and I think the more customers we do this on, the more maturity we have and the quicker we can do new implementations.

Creating value delivery routines. To realize a conceptual framework for AI, it is important to develop value-delivery models that explain how the AI solution will be brought to the customer. In this connection, informants reiterated the importance of enabling continuous improvement in the data sources and the AI solutions in different contexts, where the competence of AI providers is vital to ensure that value is perceived by the customer. Informants explained that close collaboration between the AI provider and its customers is required because better preconditions are laid down when AI solutions are applied. Thus, outcomebased business models increase opportunities to improve value creation and value capture because the provider-customer relationship is characterized by high customer engagement. R\&D investments and AI-solutions development-performed 
internally by the AI provider to acquire the technical capability - can be customized for and integrated into the customer's environment. This has been referred to as inside-out development, supporting continuous development and industrialization of AI solutions through intense customer engagement. When discussing outcome-based delivery models, the head of regional sales engagement in Sweden identified the key benefits accordingly:

We had a holistic view [when developing AI solutions], from our perspective and the delivery perspective, meaning that it is more inside-out. But we didn't always connect it to the customer's needs, outside-in, what do they need? How can it be bought? How is the value assessed? We mostly looked at it from our perspective, but when this is done, we will have an extremely powerful offering.

Informants also argued that close customer engagement creates lock-in effects, with the customer becoming dependent on the provider's ability to leverage the potential values offered by AI solutions. Thus, outcome-based delivery models are beneficial in improving customer retention measures and thus securing longterm profitability. However, intense customer collaboration has its limitations when it comes to scalability, since the AI provider will only engage with the most profitable customers and exclude those customers whose value-capture opportunities are considered less attractive or fewer. This study finds that licensing strategies support scalability to a greater extent because of the limited commitment level required by the provider. Therefore, this licensing delivery model can be beneficial when considering AI as a GPT because it has the potential to capture value from a large number of different customers. This finding is supported by the general manager in India:

You can take it off the shelf and buy it and use it. So, this is a new situation for us and for the customer. For us, we are getting additional revenue from the same efforts, from developing those algorithms, and we are also getting an entry into the customer's domain by even offering very limited solutions. So, it helps us expanding our offerings.

Thus, multiple delivery models must co-exist in order to fully capitalize on AI scalability benefits whilst simultaneously enabling continuous development. Importantly, when developing value-delivery models, the provider must deliberate on inside-out development opportunities and, at the same time, promote scalability.

Performing risk assessment. Informants spoke of several risks associated with outcome-based and licensing models, stressing the importance of assessing risks thoroughly. When discussing outcome-based risks, our informants focused primarily on customer resistance to sharing data and the development of value indicators. Insufficient or misdirected value indicators have a direct and negative impact on the provider-customer relationship, which will translate into limited data access. When discussing the licensing model, informants took the view that these risks are amplified. The provider has less control of the processes where the 
AI model is applied because it relies solely on the performance of the AI model, disclaiming responsibility for the interconnected action models. In developing and operating multiple business models simultaneously, informants asserted that the provider must ponder the risks associated with business-model cannibalization. It may well be that the licensing model is more convenient and cost effective for customers, which will lessen the attractiveness of the outcome-based model. Thus, proper consideration must be given to this when developing the business model, which calls for iteration between the three second-order activities. The business development director in the US expressed it thus:

The challenge would be to get the two things to complement each other at the same time. Because, in the Machine Learning as a service [licensing model], that is the only outcome that you have. In the outsourcing contract [outcomebased model], that is just a technique that is used, and there are many other parts that the outsourcing contract, field services, and various other pieces like that. You don't want to be in a position where you cannibalize your main service offerings.

\subsection{Developing Al business model offer}

The third theme of the analysis is about the AI business model and to design the value-capture dimension. It centers on three second-order activities, which draw on the definitions of value capture, contractual agreements, and pricing models. The three activities are framed as creating value flow structure, developing contractual agreements, and testing revenue models and are described in consecutive order.

Creating value flow structure. The foundation of value capture is how value is distributed between actors in the value creation network. It is therefore important to agree on how values are allocated between the provider and the customer with respect to the three categories of value created by AI-namely, cost efficiency, revenue increases, and business gains. In order to enable sustainable long-term relationships, win-win opportunities must be prioritized where both the provider and the customer derive benefits from the value structure. However, informants argue that such discussions must be held on a decentralized level to fully understand how AI can be utilized among customers with different preconditions. Hence, the creation of a suitable value structure relies heavily on the competence of the sales managers in the AI sphere, their ability to identify value-creation opportunities and, ultimately, their skill in converting them into a win-win value structure.

I would say that we have given the privilege to the sales team, it is up to them.

Because it all comes down to how much the customer is willing to spend, and how much they get out of it and the value that we create for each customer. There are very different selling propositions, so it all depends on how we can position well towards the customer.

Developing contractual agreements. This activity aims to create a structure of responsibility between actors in the value-creation network. As previously stated, value cannot be created by the provider on a stand-alone basis but must occur in 
collaboration with the customer. This is to ensure that the provider has access to data in the format and volume needed to create value using AI applications. Therefore, it is important to evaluate the scope of each actor's role and agree on how value will be distributed throughout the value-creation network. Consequently, the contractual agreements are dependent on the value-delivery model and its accompanying risks. However, it is vital to find common ground on data appreciation by understanding how the provider and the customer value the data in relation to the AI operation. In other words, neither data sources nor the AI solution creates value by themselves. The AI solution and its complementary assets must be evaluated on how it contributes to value creation, which will in turn lay the foundation for value capture. Informants identified this evaluation as one of the key challenges that needs to be resolved using performance indicators to increase trust.

Testing revenue models. In terms of economic compensation, pricing models are crucial elements when deciding how to establish profitable relationships with customers. To ratify the value created, informants stated that value indicators should be employed as the foundation for pricing models because they will communicate and quantify the values created in the areas of cost efficiency and revenue increases. In doing this, it is argued that business gains will play a different role in the pricing domain. As previously stated, AI can be used to refine insights on certain operational efforts and their effect on business performance. Thus, AI can be used to support the development of "proof of concept" or value indicators. Value indicators are of utmost importance because of the complexity that flows from large amounts of assorted data, which complicates the process of evaluating the effects of AI operations and network investments in general. Informants maintained that performance indicators constituted a vital element in the discussions because they have a direct impact on the trustworthiness of the AI solution and, consequently, on the customer's willingness to invest. Having said that, values in the category of business gains support the value-capture dimension through their contribution to increased transparency. The general manager in India explained how AI supports the development of value indicators:

So, then we start quantifying this, we have designed a value calculator. You put in certain inputs [to the AI model], like what's your subscriber base, what is the ARPU, what is the churn, acquisition rate, and what is the improvement in customer experience. These five parameters, you start quantifying.

In the next step, informants stated that the pricing model is dependent on the contractual agreements and for whom the values are created. This underscores the importance of aligning pricing models with contractual agreements. For instance, if the provider delivers the AI solution through an outcome-based contract-where the provider possesses the greater responsibility-AI-related benefits accrue to the provider to a greater extent than the customer. Hence, in this case, the cost-based pricing model is found to be suitable because the provider owns the focal process where it applies AI to decrease its own costs. In this case, the provider will recognize the AI-related benefits whereas the customer will not foresee any additional value. In consequence, the cost-based pricing model will be held to be more suitable. The commercial manager in the US explained the logic: 
The basic then for an offer, for a pricing offer is, if we come in with our price point, then we can demonstrate how much faster and cheaper, the net present value we can provide this benefit to you. So that is how we get to a base price, and then, typically, that is going to be a fixed fee over time, but we might have scale factors for the size of the network or the number of technologies.

However, the ultimate target when developing pricing models is to absorb the values created by AI. During the interviews, informants emphasized that AI brings extended opportunities in making the transition to value-based pricing models. The provider can utilize AI to create additional value in the areas of cost efficiency and revenue increases and, therefore, dramatically improve process performance, while the marginal costs of AI remain close to zero. The AI and automation development lead explained it thus:

We are trying to build the value minus model, we convince the operator that the cost is the software, I will be able to save X million dollars, I would like to have a portion of that. So, if there is a service, lets stick to that for the time being, then there are costs to make sure that this happens. The traditional model was that it takes Alpha X thousand dollars to build this, so I won't charge that, and we want to move away from that. We believe that AI is going to help the operator, and we should be able to help the operator.

Hence, AI providers must seek opportunities to apply the value-based pricing model in order to ensure that value is fairly distributed throughout the value-creation network. In order to do so, it is important to iterate between the three second-order activities to ensure alignment between the value structure and its correlating pricing models.

\section{Framework for business model innovation for Al solutions}

This section presents our process framework that builds on three phases-namely, identifying prerequisites for AI value creation, Matching value capture mechanisms, and developing AI business model offer. The framework (see Fig. 2) builds on the findings described above and combines phases and activities for AI business model innovation. The systematic approach of the framework development and its holistic view on the business model innovation is a major advantage.

Each phase of the framework constitutes of several activities and the first phase centers on activities needed to ensure value creation through AI. The second phase seeks to build a bridge between the value-creation and the value-capture dimensions. To connect value creation with value-capture opportunities, the task at hand is to link value-creation opportunities with the targeted customer segment. Lastly, the third phase aims to develop the value offering and the means through which value is to be captured. This requires that the previous phases are taken into account. Different value-creation opportunities mediate different approaches in the design of pricing models and contractual agreements. For each phase it is important to realize that 


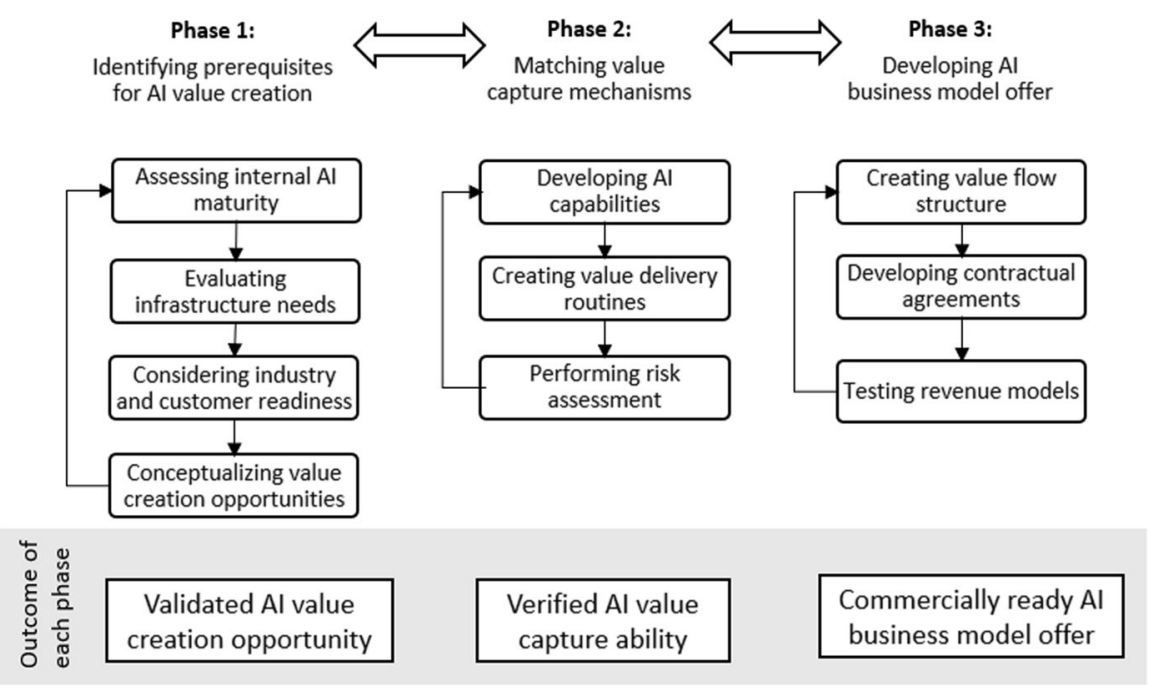

Fig. 2 Framework of business model innovation for AI solutions

in most of the cases it will be necessary to redo activities and do loops of feedback and revision to reach at the outcome of a phase.

By specifying the outcome of each phase, the framework gives a direction of the aim of the phase and this can also be used to evaluate whether a phase is completed or needs more rework before moving to the next phase (Linde et al. 2020). The outcome for the first phase is a validated AI value creation opportunity which indicted that the opportunity is both possible to develop internally but also has a value for the customers and is sellable. The outcome of the second phase is a verified AI value capture ability. This includes that the development of capabilities and routines is feasible, and that sufficient risk assessment is performed. The final outcome is the commercially ready AI business model offer. After this stage all parts are in place and especially the contractual agreements and revenue models are ready to be used in practice. However, many companies fail to revisit and redo activities from previous phases (Sjödin et al. 2020). Hence, iteration between the three phases is called for in order to ensure that the created value is captured.

\section{Conclusion}

This study extends the research on value-creation and value-capture opportunities when developing AI business models. We develop a process framework illustrating activities needed to identifying prerequisites for AI value creation, Matching value capture mechanisms, and developing AI business model offer when developing AI offerings. This section discusses the results of the study by focusing on its theoretical and practical implications. Furthermore, it presents this study's limitations and offers recommendations for future research. 


\subsection{Theoretical contribution}

Our study contributes to an understanding of the business value of AI, which is an area that needs further investigation from a theoretical point of view. This facilitates the move towards a widespread, general understanding of AI's impact on businesses. More specifically, this study presents three main findings.

Firstly, when referring to AI-related benefits, previous research has often discussed improved cost efficiency and revenue increases (Agrawal et al. 2019). This study extends previous literature and elaborates on how the implementation of AI results in overarching business gains. Thus, this study asserts the need to make a distinction between improved cost efficiency, revenue increases, and business gains. Improved cost efficiency and revenue increases are derived from operational process improvement, whereas business gains have a more far-reaching impact. This is important because they call for different approaches when shaping the value-creation activities. Thus, this distinction is central in seeking to understand how AI creates value.

Secondly, previous research points out that AI has the potential to become a GPT (Bresnahan and Trajtenberg 1995; Brynjolfsson et al. 2017; Cockburn et al. 2018), meaning that value is captured from scale. However, this is not translated into actual business model concepts that enable AI to become a GPT. To fill this gap, our study presents a framework to describe how AI, as a GPT, impacts the value-creation and value-capture dimensions. In addition, this research highlights the need to evaluate two parameters when modeling the value-capture dimension-namely, scalability and continuous improvement. However, in order to facilitate both scalability and continuous improvement, it is necessary to develop multiple business-model concepts that support these two parameters.

Thirdly, we find that the development of value-based pricing models is required in order to fully capitalize on AI. This is due to the low marginal costs of AI solutions and the potential to create massive cost efficiency improvements and revenue increases (Choi et al. 2010). However, this study stresses that value creation is highly dependent on operational action models, developed to act on insights provided by AI. In other words, it is important to consider surrounding elements when designing the business model and to establish how value is captured. Therefore, cost-based pricing models are held to be suitable if the surrounding elements center on cost reduction rather than revenue increases or business gains. In addition, we found that the design of pricing models depend on how contractual agreements are formed. For instance, offering solutions that include both action models and AI employing outcome-based arrangements, and that are characterized by high customer interaction, are found to increase value-capture opportunities for individual customers. Indeed, high customer interaction and trust are paramount when establishing the value structure by designing value indicators, as Sjödin et al. (2020) have found. However, when offering AI solutions separately from action models using a licensing approach, value-capture opportunities are limited by ownership issues. This calls for greater discussion on who owns the results of the operation where action models act on information provided by AI. 


\subsection{Practical implications}

Our study carries practical implications for the many firms who aim to implement and take advantage of the seemingly massive potential that flows from AI. Its impact on cost efficiency and revenue increases has been generating increased interest across a wide range of industries. However, due to a limited understanding of AI and its implications on the development of business models, few companies have managed to implement AI successfully. In this regard, our study contributes to a practical understanding of AI by designing a process framework, showcasing the implementation of AI. Its three phases guide practitioners when implementing AI by describing the activities needed to identifying prerequisites for AI value creation, Matching value capture mechanisms, and developing AI business model offer. Hence, the three phases and their underlying activities support managers and increase company awareness of how AI can be successfully implemented. Furthermore, the framework provided is applicable to all firms that seek to implement AI. However, when adopting the view of AI as a GPT, extra caution should be directed to evaluating the needed infrastructure elements. This overarching cloud infrastructure is vital to ensure that the AI provider has access to sufficient data in the right format. In other words, the framework's practical implications rely heavily on the firm's ability to access and manage large data sets.

In addition, this study's view of AI as a GPT and its contribution to how firms can realize its full potential, implies increased opportunities to capture value from a larger scale. The co-existence of multiple business models fosters opportunities to cross industry boundaries and reach a wider customer base. This points not only to financial benefits for AI providers but also to increased opportunities for downstream actors to benefit from AI. However, there is a need for iteration between the three phases because new customer segments or application areas call for various value-creation opportunities, different prerequisites in the value-creation dimension, and also diverse opportunities to capture the value created. Therefore, practitioners should be aware that the framework presented represents a continuous process and that the business models must be regularly refined. This is to ensure that managers close the gap between changes in the surrounding environment (e.g., technological advances or shifting demand) and the business-model concept. Moreover, the framework can be used not only to develop and establish new business-model concepts but also to refine existing business models.

\subsection{Limitations and future research}

This study has its limitations, and AI's impact on businesses is still a relatively unexplored subject that calls for further research. This study hopes to inspire future research by directing researchers to cover three limitations. Firstly, this study is limited by its scope, which placed emphasis on a qualitative approach using a single case study. Background information on the case company and its surrounding environment (i.e., characteristics of the telecom industry) is described. However, the results should be handled with caution if surrounding elements are different. Therefore, future research should consider transferability by conducting quantitative research, preferably by combining a deductive and inductive reasoning in a flexible 
pattern matching approach (Bouncken and Tiberius (2021). Secondly, this study is limited by the fact that its collected data are sourced from a single AI provider and, consequently, the customer's perspective might be sidelined. Therefore, more qualitative research in which the customer's perspective is taken fully into consideration is called for. Lastly, this study identified the need for AI providers to develop multiple business models and operate them simultaneously. Yet, this approach could entail substantial risks of business-model cannibalization, which might hamper both value-creation and value-capture opportunities. Therefore, future research should investigate how this risk can be mitigated by exploring how multiple business models could co-exist. Preferably, this can be achieved by creating a framework that explicitly describes how different business models' interplay.

Funding Open access funding provided by Lulea University of Technology.

Open Access This article is licensed under a Creative Commons Attribution 4.0 International License, which permits use, sharing, adaptation, distribution and reproduction in any medium or format, as long as you give appropriate credit to the original author(s) and the source, provide a link to the Creative Commons licence, and indicate if changes were made. The images or other third party material in this article are included in the article's Creative Commons licence, unless indicated otherwise in a credit line to the material. If material is not included in the article's Creative Commons licence and your intended use is not permitted by statutory regulation or exceeds the permitted use, you will need to obtain permission directly from the copyright holder. To view a copy of this licence, visit http://creativecommons.org/licen ses/by/4.0/.

\section{References}

Agrawal A, Gans JS, Goldfarb A (2019) Artificial intelligence: the ambiguous labor market impact of automating prediction. J Econ Perspect 33(2):31-50

Barney JB (1999) How a firm's capabilities affect boundary decisions. MIT Sloan Manage Rev 40(3):137

Bouncken RB, Tiberius V (2021) Legitimacy processes and trajectories of co-prosumption services: insights from coworking spaces. J Service Res. https://doi.org/10.1177/10946705211050208

Bouncken RB, Kraus S, Roig-Tierno N (2021) Knowledge-and innovation-based business models for future growth: digitalized business models and portfolio considerations. RMS 15(1):1-14

Bresnahan TF, Trajtenberg M (1995) General purpose technologies 'engines of growth'? J Econ 65(1):83-108

Brock JK-U, Von Wangenheim F (2019) Demystifying AI: What digital transformation leaders can teach you about realistic artificial intelligence. Calif Manage Rev 61(4):110-134

Brynjolfsson E, Rock D, Syverson C (2017) Artificial intelligence and the modern productivity paradox: a clash of expectations and statistics. Technical report, Nat Bur Econ Res

Chesbrough H (2007) Business model innovation: it's not just about technology anymore. Strategy Leadership 35(6):12-17

Chesbrough H, Rosenbloom RS (2002) The role of the business model in capturing value from innovation: evidence from xerox corporation's technology spin-off companies. Ind Corp Change 11(3):529-555

Choi JP, Fershtman C, Gandal N (2010) Network security: vulnerabilities and disclosure policy. J Ind Econ 58(4):868-894

Cockburn IM, Henderson R, Stern S (2018) The impact of artificial intelligence on innovation. Technical report, Nat Bur Econ Res

Gambardella A, McGahan AM (2010) Business-model innovation: general purpose technologies and their implications for industry structure. Long Range Plann 43(2-3):262-271

Gassmann O, Frankenberger K, Csik M (2013) The St Gallen business model navigator

Gioia DA, Corley KG, Hamilton AL (2013) Seeking qualitative rigor in inductive research: notes on the Gioia methodology. Organ Res Methods 16(1):15-31 
Hinterhuber A (2008) Customer value-based pricing strategies: why companies resist. J Bus Strat 29(4):41-50

Hou J, Neely A (2018) Investigating risks of outcome-based service contracts from a provider's perspective. Int J Prod Res 56(6):2103-2115

Kakatkar C, Bilgram V, Füller J (2019) Innovation analytics: leveraging artificial intelligence in the innovation process. Bus Horizons

Kaplan A, Haenlein M (2019) Siri, siri, in my hand: Who's the fairest in the land? On the interpretations, illustrations, and implications of artificial intelligence. Bus Horizons 62(1):15-25

Kok JN, Boers E, Kosters WA, Van der Putten P, Poel M (2009) Artificial intelligence: definition, trends, techniques, and cases. Artif Intell 1:270-299

Kraus S, Roig-Tierno N, Bouncken RB (2019) Digital innovation and venturing: an introduction into the digitalization of entrepreneurship. RMS 13(3):519-528

Laudien SM, Pesch R (2019) Understanding the influence of digitalization on service firm business model design: a qualitative-empirical analysis. RMS 13(3):575-587

LeCun Y, Bengio Y, Hinton G (2015) Deep learning. Nature 521(7553):436-444

Lee J, Suh T, Roy D, Baucus M (2019) Emerging technology and business model innovation: the case of artificial intelligence. J Open Innov Technol Market Complex 5(3):44

Lincoln YS (2007) Naturalistic inquiry. The Blackwell Encyclopedia of Sociology

Linde L, Sjödin D, Parida V, Gebauer H (2020) Evaluation of digital business model opportunities: a framework for avoiding digitalization traps. Res Technol Manag 64(1):43-53

Liozu SM, Hinterhuber A, Boland R, Perelli S (2012) The conceptualization of value-based pricing in industrial firms. J Revenue Pric Manag 11(1):12-34

Lungu MF (2018) Achieving strategic agility through business model innovation the case of telecom industry. In Proceedings of the International Conference on Business Excellence, 12(1): 557-567

Moeen M, Agarwal R (2017) Incubation of an industry: heterogeneous knowledge bases and modes of value capture. Strateg Manage J 38(3):566-587

Muhic M, Bengtsson L (2021) Dynamic capabilities triggered by cloud sourcing: a stage-based model of business model innovation. RMS 15(1):33-54

Nylund PA, Ferras-Hernandez X, Brem A (2020) Automating profitably together: Is there an impact of open innovation and automation on firm turnover? Rev Manag Sci 14(1):269-285

Pisano GP, Teece DJ (2007) How to capture value from innovation: Shaping intellectual property and industry architecture. Calif Manage Rev 50(1):278-296

Plastino E, Purdy M (2018) Game changing value from artificial intelligence: eight strategies. Strategy Leadership 46(1):16-22

Ransbotham S, Khodabandeh S, Fehling R, Lafountain B, Kiron D (2019) Winning with AI. Technical Report

Shaw J, Rudzicz F, Jamieson T, Goldfarb A (2019) Artificial intelligence and the implementation challenge. J Med Internet Res 21(7):e13659

Simon H, Butscher SA, Sebastian K-H (2003) Better pricing processes for higher profits. Bus Strateg Rev 14(2):63-67

Sjödin D, Parida V, Jovanovic M, Visnjic I (2020) Value creation and value capture alignment in business model innovation: a process view on outcome-based business models. J Prod Innov Manag 37(2): $158-183$

Teece DJ (2018) Profiting from innovation in the digital economy: Enabling technologies, standards, and licensing models in the wireless world. Res Policy 47(8):1367-1387

Thomas DW, Burns J, Audette J, Carroll A, Dow-Hygelund C, Hay M (2016) Clinical development success rates 2006-2015. BIO Ind Anal 1:16

Thornhill A, Saunders M, Lewis P (2009) Research methods for business students. Prentice Hall, London

Valter P, Lindgren P, Prasad R (2018) The consequences of artificial intelligence and deep learning in a world of persuasive business models. IEEE Aero El Sys Mag 33(5-6):80-88

Visnjic I, Jovanovic M, Neely A, Engwall M (2017) What brings the value to outcome-based contract providers? Value drivers in outcome business models. Int J Prod Econ 192:169-181

Zhuang Y-T, Wu F, Chen C, Pan Y-H (2017) Challenges and opportunities: from big data to knowledge in AI 2.0. Front Inform Tech El 18(1):3-14

Publisher's Note Springer Nature remains neutral with regard to jurisdictional claims in published maps and institutional affiliations. 